\title{
Development of transparent microwell arrays for optical monitoring and dissection of microbial communities
}

Michelle Halsted, Jared L. Wilmoth, Paige A. Briggs, Ryan R. Hansen, Dayrl P. Briggs, Andrea C. Timm, and Scott T. Retterer

Citation: Journal of Vacuum Science \& Technology B, Nanotechnology and Microelectronics: Materials, Processing, Measurement, and Phenomena 34, 06 KI03 (2016); doi: 10.1116/1.4962739

View online: http://dx.doi.org/10.1116/1.4962739

View Table of Contents: http://avs.scitation.org/toc/jvb/34/6

Published by the American Vacuum Society

\section{Articles you may be interested in}

Milling a silicon nitride membrane by focused ion beam

Journal of Vacuum Science \& Technology B, Nanotechnology and Microelectronics: Materials, Processing, Measurement, and Phenomena 34, 062201 (2016); 10.1116/1.4963895

PMMA removal selectivity to polystyrene using dry etch approach Journal of Vacuum Science \& Technology B, Nanotechnology and Microelectronics: Materials, Processing, Measurement, and Phenomena 34, 061802 (2016); 10.1116/1.4964881

Displacement Talbot lithography nanopatterned microsieve array for directional neuronal network formation in brain-on-chip

Journal of Vacuum Science \& Technology B, Nanotechnology and Microelectronics: Materials, Processing, Measurement, and Phenomena 34, 06 KI02 (2016); 10.1116/1.4961591

Prediction of surface topography due to finite pixel spacing in FIB milling of rectangular boxes and trenches Journal of Vacuum Science \& Technology B, Nanotechnology and Microelectronics: Materials, Processing, Measurement, and Phenomena 34, 061803 (2016); 10.1116/1.4967249

Electron beam lithography patterned hydrogen silsesquioxane resist as a mandrel for self-aligned double patterning application

Journal of Vacuum Science \& Technology B, Nanotechnology and Microelectronics: Materials, Processing, Measurement, and Phenomena 34, 061601 (2016); 10.1116/1.4963194

Effects of aging on nanoscale planar metal-insulator-metal tunnel junctions Journal of Vacuum Science \& Technology B, Nanotechnology and Microelectronics: Materials, Processing, Measurement, and Phenomena 34, 062203 (2016); 10.1116/1.4967786

Contact Hiden Analytical for further details: w www.HidenAnalytical.com E info@hiden.co.uk CLICK TO VIEW our product catalogue

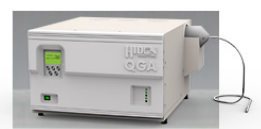

Gas Analysis

dynamic measurement of reaction gas streams catalysis and thermal analysis molecular beam studies molecular beam studies , dissolved spectes probes s

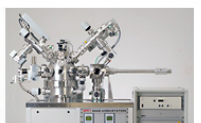

Surface Science

UHVTPD

SIMS

end point detection in ion beam etch elemental imaging - surface mapping

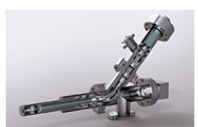

Plasma Diagnostics

plasma source characterization etch and deposition process reaction kinetic studies analysis of neutral and radical species

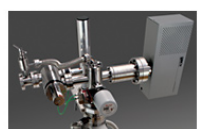

Vacuum Analysis , partial pressure measurement and control of process gases reactive sputter process control vacuum diagnostics , vacuum coating process monitoring 


\title{
Development of transparent microwell arrays for optical monitoring and dissection of microbial communities
}

\author{
Michelle Halsted \\ The Bredesen Center, The University of Tennessee, Knoxville, Tennessee 37996 \\ Jared L. Wilmoth \\ Biosciences Division, Oak Ridge National Laboratory, Oak Ridge, Tennessee 37831 \\ Paige A. Briggs \\ Mechanical Engineering, The University of Alabama, Tuscaloosa, Alabama 35487
}

Ryan R. Hansen

Chemical Engineering, Kansas State University, Manhattan, Kansas 66506

Dayrl P. Briggs

Center for Nanophase Materials Sciences, Oak Ridge National Laboratory, Oak Ridge, Tennessee 37831

Andrea C. Timm

Biosciences Division, Oak Ridge National Laboratory, Oak Ridge, Tennessee 37831

Scott T. Retterer ${ }^{\mathrm{a})}$

Biosciences Division, Oak Ridge National Laboratory, Oak Ridge, Tennessee 37831; The Bredesen Center, University of Tennessee, Knoxville, Tennessee 37996; and Center for Nanophase Materials Sciences, Oak Ridge National Laboratory, Oak Ridge, Tennessee 37831

(Received 24 June 2016; accepted 29 August 2016; published 29 September 2016)

\begin{abstract}
Microbial communities are incredibly complex systems that dramatically and ubiquitously influence our lives. They help to shape our climate and environment, impact agriculture, drive business, and have a tremendous bearing on healthcare and physical security. Spatial confinement, as well as local variations in physical and chemical properties, affects development and interactions within microbial communities that occupy critical niches in the environment. Recent work has demonstrated the use of silicon based microwell arrays, combined with parylene lift-off techniques, to perform both deterministic and stochastic assembly of microbial communities en masse, enabling the high-throughput screening of microbial communities for their response to growth in confined environments under different conditions. The implementation of a transparent microwell array platform can expand and improve the imaging modalities that can be used to characterize these assembled communities. Here, the fabrication and characterization of a next generation transparent microwell array is described. The transparent arrays, comprised of SU-8 patterned on a glass coverslip, retain the ability to use parylene lift-off by integrating a low temperature atomic layer deposition of silicon dioxide into the fabrication process. This silicon dioxide layer prevents adhesion of the parylene material to the patterned SU-8, facilitating dry lift-off, and maintaining the ability to easily assemble microbial communities within the microwells. These transparent microwell arrays can screen numerous community compositions using continuous, high resolution, imaging. The utility of the design was successfully demonstrated through the stochastic seeding and imaging of green fluorescent protein expressing Escherichia coli using both fluorescence and brightfield microscopies. (C) 2016 American Vacuum Society.
\end{abstract}

[http://dx.doi.org/10.1116/1.4962739]

\section{INTRODUCTION}

Microbial communities shape the development of critical and unique environments, dramatically impacting agriculture, climate change, and human health. Naturally occurring microbial assemblages are expected to exist in a wide variety of spatially distinct microenvironments or niches. Adequately assessing these communities may not be possible in homogeneous batch-model systems. The ability to understand, optimize, and direct the activity of complex microbial communities that develop in spatially confined environments holds

a)Electronic mail: rettererst@ornl.gov significant potential for biological and bioengineering research. Methods for performing high-throughput screening are essential for understanding the role of community composition and structure on both function and persistence. We have recently developed and described a microwell array platform that allows both deterministic and stochastic assembly of microbial communities for large scale monitoring and testing. ${ }^{1}$ Here, we describe the fabrication and testing of an enhanced transparent version of that platform that retains the functionality of its predecessor, while dramatically expanding the imaging modalities that can be used to monitor the platform and improve fluidic access to the chemical microenvironment. 
Community development and function are heavily influenced by member abundance, confinement, and niche connectivity; thus, the complexities of this vast parameter space require the implementation of high-throughput and highcontent screening platforms. ${ }^{1-3}$ Nanofabrication offers not only a method for high-throughput screening, but also control over bacterial microenvironments. ${ }^{4}$ Microwell array platforms enable the en masse study of microbial communities, both enhancing and complementing conventional omics approaches to community characterization. Previous work has shown that microwell arrays retain the ability to address both spatial organization and environmental diversity regarding nutrient exchange within microbial communities. ${ }^{1,5}$ These platforms require limited or no connections to flow control systems and are highly compatible with conventional microscopes. Other high-throughput approaches include microfluidic droplet systems, where bacteria are isolated within a defined aqueous phase. ${ }^{6-9}$ Although many automated microdroplet systems are primarily used for screening a single species of bacteria for interactions with antibiotics, ${ }^{7,10,11}$ a microdroplet approach has also been demonstrated to be useful for community studies. ${ }^{8}$ Microdroplet systems and well plates alike often utilize liquid media, which may not be suitable for the growth and maintenance of some bacteria, ${ }^{5}$ making the solid microwell array a desirable alternative in such cases.

Monitoring discrete spatial organization of cells within microbial communities is essential to reach a better understanding of higher-order community function. ${ }^{2}$ The microwell array platform introduced by Hansen $e t$ al. explores the impact of well diameter, well depth, and environmental conditions on the assembly of microbial communities. This array platform allows for both stochastic and deterministic seeding of microbial cells into microwells, facilitated by the use of a parylene stencil that enables dry lift-off. ${ }^{1}$ Parylene (poly-para-xylylene) is a plastic film commonly used in biomedical devices. ${ }^{12-14}$ After deposition on the substrate, select areas of the parylene, aligned with the microwells, are removed by etching. Subsequently, cells are added to cover the entire chip. When the parylene stencil is peeled away, only those cells that seeded through the holes in the parylene (i.e., within the microwells) remain behind. The microwell array described in Hansen et al. was made from an optically opaque silicon substrate. These arrays can then be capped with an agar-covered glass coverslip to maintain nutrient flux for growing cells within the arrays. This configuration requires the use of a longer working distance and lower numerical aperture objective to image through the transparent materials of both the coverslip and agar layer, as opposed to the transparent SU-8 base (Fig. 1). While fluorescent cells can be observed during image analysis on this platform, cells cannot be imaged using standard brightfield microscopy. Imaging through an agar layer is also not optimal for longer duration growth studies and time-lapse imaging since evaporation can lead to shrinking of the agar and movement of the sample out of the focal plane. This movement can significantly alter both the intensity and resolution of collected images. (a)

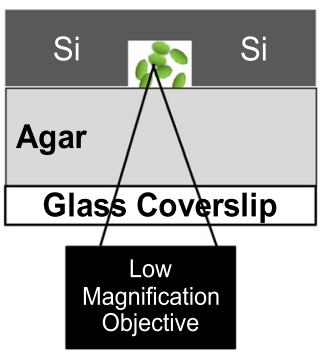

(b)

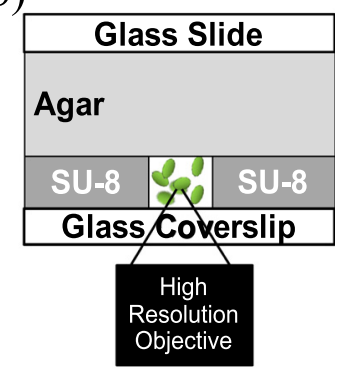

FIG. 1. (Color online) Schematic representation of the microwell assembly and imaging. (a) A silicon microwell array is oriented with the well opening facing down on an inverted scope. The wells are imaged through a glass coverslip and a hydrated layer of agar. (b) A transparent microwell array allows for imaging directly through the coverslip-substrate using higher resolution, shorter working distance objectives. Transmitted light, brightfield, and phase contrast imaging can be performed with the transparent microwells.

The use of a transparent material for the base microwell array enables high-resolution imaging of bacterial communities within each microwell. Imaging through a transparent base allows for a significant decrease in the amount of material between the objective lens and the cells growing within the microwells and enables high resolution imaging using both brightfield and fluorescence microscopies. Here we describe a simple, yet elegant fabrication process for creating our transparent microwell array platform. We outline the design and utility of the newly developed transparent microwell array and demonstrate its suitability for high resolution and high-throughput screening of microbial assemblages.

\section{EXPERIMENT}

\section{A. Fabrication of microwell arrays}

Fabrication of the transparent microwells was carried out using a combination of SU-8 lithography, atomic layer deposition (ALD), photolithography, and reactive ion etching (Fig. 2). Transparent microwell arrays were created by first patterning SU-8 photoepoxy on $120 \mu \mathrm{m}$ thick glass cover slips. To improve adhesion of the SU-8 to the glass, the coverslips were heated for at least $1 \mathrm{~h}$ on a hot plate at $180^{\circ} \mathrm{C}$. This dehydrated the glass, reducing moisture at the interface between the spun-cast SU-8 and the glass coverslip. SU-8 2005 (MicroChem Corp., Westborough, MA) was spun-cast onto the coverslip, with the spin-speed and duration chosen according to the manufacturer's recommendations for the desired thickness. SU-8 2005 spun at $3000 \mathrm{rpm}$ for $45 \mathrm{~s}$ yielded films approximately $6 \mu \mathrm{m}$ thick. A soft bake at $95^{\circ} \mathrm{C}$ was performed for $2 \mathrm{~min}$ prior to exposure for $\sim 5.4 \mathrm{~s}$ in a Quintel UV Contact Aligner (Rochester, NY) at a power of $\sim 10 \mathrm{~mW} / \mathrm{cm}^{2}(365 \mathrm{~nm})$.

After exposure, the sample was placed on a $65^{\circ} \mathrm{C}$ hot plate for $1 \mathrm{~min}$, and then a $95^{\circ} \mathrm{C}$ hot plate for $3 \mathrm{~min}$. After removal from the hotplate, SU-8 developer (MicroChem Corp., Westborough, MA) was pooled on the sample ("puddle develop") for $70 \mathrm{~s}$, rinsed with additional SU-8 developer, isopropyl alcohol, and then dried with $\mathrm{N}_{2}$.

Patterning of the parylene stencil onto SU-8 presents some unique challenges not encountered in the previously 
(a)

Glass Coverslip

(b)

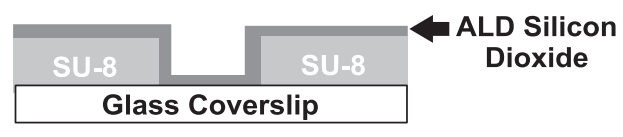

(c)

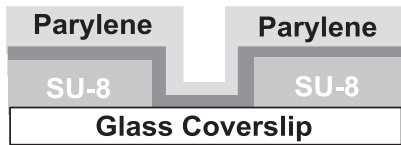

NFR

(d)

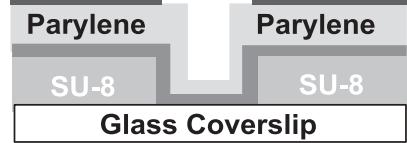

(e)

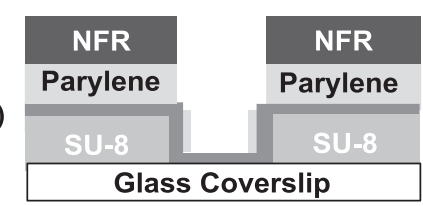

FIG. 2. Fabrication of transparent microwell arrays is carried out by (a) first performing a dehydration bake on a coverslip substrate (b) SU-8 photolithography is used to define the microwell geometry and is followed by the deposition of a low-temperature ALD silicon dioxide film. (c) Parylene deposition is followed by (d) the photopatterning of an aligned negative resist photomask. (e) An oxygen plasma etch is used to remove the exposed parylene, leaving parylene only on the well side walls. Diagram is not drawn to scale.

used silicon well fabrication process. Parylene adheres to the patterned SU-8, unlike the silicon dioxide present on the surface of the silicon arrays. To prevent adhesion to the patterned SU-8, we utilized a low-temperature ALD process for silicon dioxide deposition. The low-temperature plasma ALD was carried out in an Oxford FlexAL Atomic Layer Deposition System (Oxford Instruments, Abingdon, UK). The low-temperature process, carried out at $150^{\circ} \mathrm{C}$, is critical to avoiding reflow and deformation of the SU- 8 microwells. The ALD process utilizes a bisdiethylaminosilane precursor. Each ALD cycle consisted of a dose, purge, and plasma treatment step. Dosing of precursor was carried out under flow of argon and oxygen, 100 and $1 \mathrm{sccm}$, respectively, at $80 \mathrm{mTorr}$ for $800 \mathrm{~ms}$. The dosing step was followed by a purge under the same conditions with no precursor for $5 \mathrm{~s}$. Purging was followed by treatment with an oxygen plasma $\left(\mathrm{O}_{2}: 60 \mathrm{sccm}\right.$, 15 mTorr). Plasma gas stabilization was carried out for $2 \mathrm{~s}$ and followed by $3 \mathrm{~s}$ of $\mathrm{O}_{2}$ plasma treatment at $250 \mathrm{~W}$ for $3 \mathrm{~s}$. Postplasma stabilization (Ar: $100 \mathrm{sccm}, 80$ mTorr) was carried out for $1.5 \mathrm{~s}$ before repeating the cycle. Silicon deposition was approximately $1.2 \AA /$ cycle for each $12.3 \mathrm{~s}$ cycle. Fifty cycles were sufficient to coat wells with silicon dioxide and prevent parylene adhesion.

Parylene was deposited on the samples using a Labcoter 2 parylene Deposition Unit Model PDS 2010 (Specialty Coating Systems, INC, Indianapolis, IN). Parylene N, with a thickness of $1.0 \mu \mathrm{m}$ (with a load of $6.0 \pm 0.5 \mathrm{~g}$ ), reduced the risk of parylene tearing during lift-off. To define the photoresist mask for parylene etching and removal, an approximately $3.5 \mu \mathrm{m}$ layer of negative tone photoresist is spun-cast onto the samples. The use of a negative tone resist is critical to this process in order to allow complete removal of the resist from the interior of the microwells during development. A positive tone resist would not likely receive adequate exposure within higher aspect ratio wells to allow adequate removal. By use of a contact aligner, the sample is aligned under a slightly oversized version of the microwell array mask and exposed. Following lithography, an oxygen plasma etch (Oxford Instruments, Abingdon, UK) $\left(60 \mathrm{mTorr}, 20^{\circ} \mathrm{C}\right.$, $100 \mathrm{sccm} \mathrm{O}$, $10 \mathrm{~W}$ RF, $2000 \mathrm{~W}$ inductively coupled plasma) is used to remove parylene from the bottom of the wells. As indicated in Fig. 2(e), a parylene side wall is expected to remain within the microwell as the oxygen plasma etch is anisotropic with the etch rate in the vertical direction being faster than the horizontal etch rate. Complete removal of the parylene would be possible by extending the duration of the etch process. With proper parylene removal around the corners of the microwell, the residual parylene does not affect lift-off.

\section{B. Bacterial culture}

Fluorescent Escherichia coli K-12 was used to study bacteria seeding behavior in the microwell array platform. The strains were established by chromosomal insertion of the genes encoding GFP using a Tn7 site specific transposon system as described previously. ${ }^{1}$ Post transformation, this strain was stored in glycerol stocks at $-80^{\circ} \mathrm{C}$. Working cultures were maintained on lysogeny broth (LB) agar plates $(10 \mathrm{~g}$ $\mathrm{NaCl}, 5 \mathrm{~g}$ yeast, $10 \mathrm{~g}$ bactotryptone, and $15 \mathrm{~g}$ agar per liter). Methods for the seeding of bacteria within the microwell arrays followed those outlined in Hansen et al. and are briefly described here. E. coli K-12 expressing GFP was grown to the logarithmic phase in liquid LB media in a sterile $15 \mathrm{ml}$ centrifuge tube $\left(225 \mathrm{rpm}, 37^{\circ} \mathrm{C}\right)$, harvested by centrifugation, and resuspended in LB media to an optical density of 0.1 in a $2 \mathrm{ml}$ Eppendorf tube. A $100 \mu \mathrm{l}$ aliquot of this cell suspension was then pipetted onto the transparent microwell arrays, following an initial addition of $500 \mu \mathrm{g} \mathrm{ml}^{-1}$ bovine serum albumin (BSA) diluted in $1 \times$ phosphate buffered saline (PBS). ${ }^{1}$ Excess BSA-PBS solution was dried under a stream of $\mathrm{N}_{2}$ before adding the cell culture. Upon addition of the cell suspension, the assembly was incubated in a humidified plastic box for $1 \mathrm{~h}$ under standard conditions. After $1 \mathrm{~h}$, excess cell suspension was removed by contacting the edges of the underlying coverslip with a Kimwipe, taking care to avoid complete drying of the microwells. The parylene film was then removed using a small piece of Scotch ${ }^{\mathrm{TM}}$ tape and tweezers, removing bacteria that did not seed into the wells. Following the removal of parylene and unseeded cells, an LB 2\% agar layer was cast on a separate glass coverslip of the same dimensions and placed on top of the microwell arrays to provide nutrient flux to growing cells over an $18 \mathrm{~h}$ period, as described previously. ${ }^{1}$ The arrays were then imaged using epifluorescence and brightfield illumination.

\section{Sample imaging}

A Nikon Eclipse Ti-U inverted confocal microscope equipped with an ORCA-Flash 4.0 camera (Hamamatsu) was used to perform optical imaging of the microwell arrays 
(a)

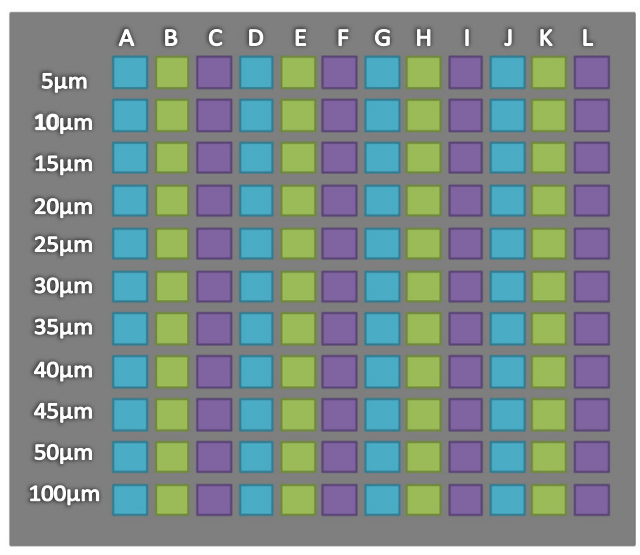

$400 \mu \mathrm{m}$

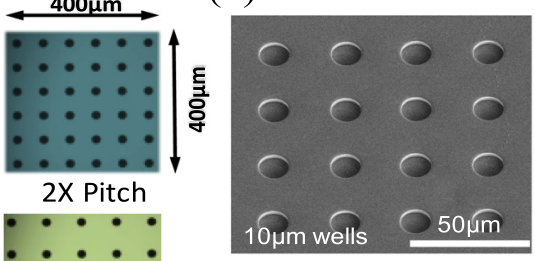

(c)

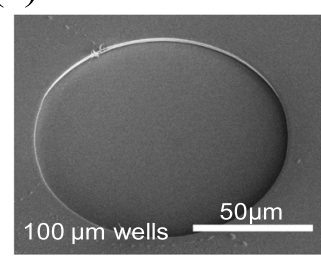

FIG. 3. (Color online) Microwell array chip layout (a) Individual coverslips contained arrays of microwells ranging from 5 to $100 \mu$ m. The pitch was varied by $2 \times, 3 \times$, and $4 \times$ of the well diameter. [(b) and (c)] SEM images of microwells following removal of the parylene layer show clean lift-off.

after cell seeding. Brightfield images were captured under transmitted light illumination to visualize E. coli cells. Fluorescent images were taken using fluorescein isothiocyanate filter sets to only show those bacteria expressing GFP. Prior to growth studies, imaging conditions were optimized such that no detectable photobleaching occurred, and to adjust the excitation source to $25 \%$, a neutral density filter was used. Under this setting, no significant bleaching of the cells was observed.

An FEI Novalab 600 Dual-Beam System was used to collect scanning electron microscopy (SEM) images of the microwell arrays at different points in the fabrication process to observe structural features of the microwell arrays after etching and parylene removal. Images were acquired while operating at $2 \mathrm{keV}$. Samples were imaged with a $30^{\circ}$ tilt to provide images of the well sidewalls.

\section{RESULTS AND DISCUSSION}

The transparent microwell arrays created with SU-8 $2005 \mathrm{had}$ an average depth of $5.9 \pm 0.3 \mu \mathrm{m}$ (KLA Tencor Profilometer, Milpitas, CA), with well diameters ranging from 5 to $100 \mu \mathrm{m}$. The array consisted of 11 rows, with diameters increasing from 5 to $50 \mu \mathrm{m}$ in increments of $5 \mu \mathrm{m}$ with an additional set of $100 \mu \mathrm{m}$ wells. As shown in previous work, this larger diameter allows for low variation in community structure and can be used as a reference during experiments. ${ }^{1}$ The 12 columns varied by pitch, where pitch is defined as the distance between each well with respect to number of equivalent well diameters, i.e., $5 \mu \mathrm{m}$ well with $2 \times$ pitch would have a spacing of $10 \mu \mathrm{m}$ between each well (Fig. 3).

The transparent microwell arrays have been successfully fabricated with parylene lift-off capabilities. Figures 3(b) and 3(c) show a scanning electron micrographs patterned microwell following parylene lift-off. The ability to seed microbes into these wells was successfully demonstrated with E. coli expressing GFP (Fig. 4). Microbial growth was observed within the microwells, and was successfully imaged over a period of $12 \mathrm{~h}$ microscopy (Fig. 4).
Additionally, Fig. 4 illustrates the high resolution imaging capability of the transparent microwell array for both fluorescence and brightfield microscopy. The ability to capture brightfield, high resolution, micrographs of the microwell arrays is a critical advantage to the study of microbial communities as bacteria do not need to be tagged with fluorescent labels or modified to express fluorescent proteins for observation and monitoring of growth and development.

Stochastic seeding enables the screening of unique microbial communities across numerous member compositions (a)

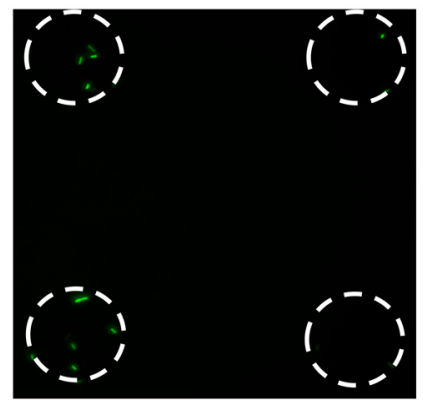

(c)

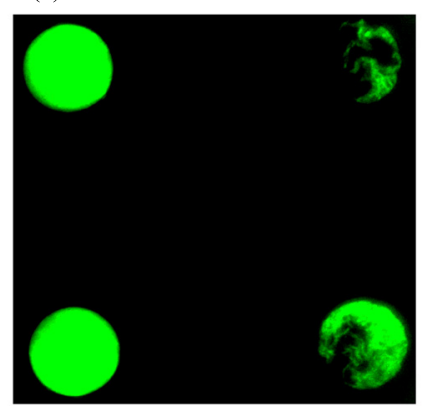

(b)

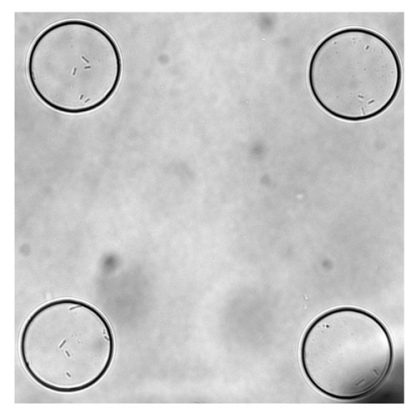

(d)

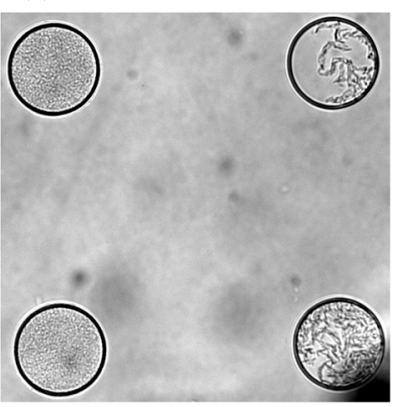

FIG. 4. (Color online) Images of E. coli cells expressing green fluorescent protein (GFP) seeded within four $45 \mu \mathrm{m}$ diameter wells at $40 \times$ magnification, after parylene lift-off, using (a) epifluorescence, time zero; (b) brightfield, time zero; (c) epifluorescence after $18 \mathrm{~h}$; and (d) brightfield after $18 \mathrm{~h}$. Both fluorescence and brightfield image sets confirm that E. coli cells remain vitable over an $18 \mathrm{~h}$ period inside the wells, confirming that this new platform is compatible with active, growing cells. 
(a)

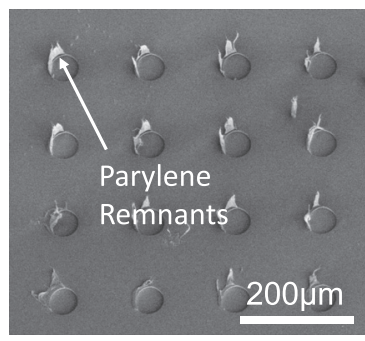

(b)

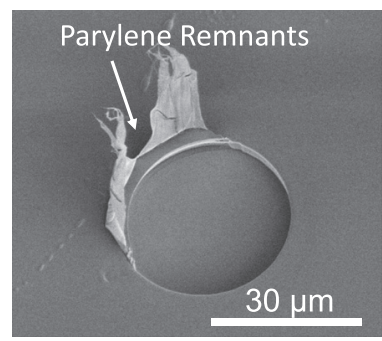

(c)

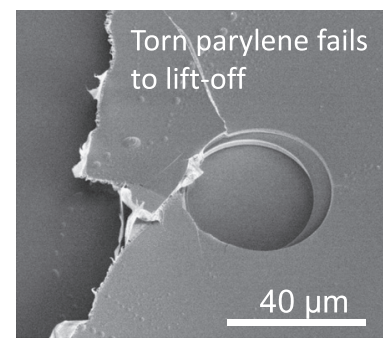

FIG. 5. Misalignment of parylene lift-off layer with SU-8 wells. [(a) and (b)] Remnants of parylene left behind following lift-off and (c) tearing off of larger pieces of parylene can result from incomplete poor alignment and incomplete etching.

and environmental parameters, and is achieved through use of the parylene lift-off layer. ${ }^{1}$ However, parylene adheres to SU-8 epoxy, thus to facilitate lift-off, a low temperature plasma assisted atomic layer deposition of silicon dioxide was introduced as a separation layer between the SU-8 and parylene. Without the silicon dioxide layer, attempts to remove parylene from SU-8 required forces that ultimately break the $120 \mu \mathrm{m}$ thick glass coverslip. While lift-off is facilitated by the presence of the ALD layer, misalignment of the negative photoresist etch mask did lead to insufficient removal of parylene in some cases. Parylene deposition on three-dimensional wells requires an additional alignment and photolithography step to define the photoresist etch mask for the oxygen plasma etching process. This etch process essentially breaks the connection between the parylene on the substrate surface and the parylene within the microwells. Accurate alignment is essential to cleanly breaking this connection and preventing tearing of the parylene during lift-off. Failure to properly align the SU-8 microwells with the microwells of the photoresist etch mask results in parylene tearing that can lead to microscopic remnants of parylene remaining in the wells after lift-off [Figs. 5(a) and 5(b)] or tearing of the parylene, which leaves larger areas unremoved [Fig. 5(c)].

Direct etching of the glass or quartz substrate was considered for the fabrication of transparent microwell arrays. This approach was unsuccessful due to poor stability of the parylene layer during etching and limited etch rates of the quartz substrate. Additionally, etching prior to parylene deposition, or in the absence of parylene, yielded wells with rough surfaces. This limited the optical properties of the transparent base and resulted in an undesirable environment to grow and monitor bacteria.

\section{SUMMARY AND CONCLUSIONS}

The transparent microwell arrays, comprised of SU-8 patterned on $120 \mu \mathrm{m}$-thick glass coverslips and a parylene stencil for dry lift-off, were successfully fabricated and used to seed and image bacteria. These arrays not only expand the modes of imaging that can be used to monitor microbial growth but also improve the resolution of such imaging techniques by reducing the working distance to the sample, decreasing the number of layers of material that must be imaged through, and providing a more stable platform. Transparent arrays retain the ability to use parylene lift-off for cell seeding by integrating a low temperature ALD silicon dioxide layer into the fabrication process. This prevents adhesion of the parylene material to the patterned SU-8, facilitating dry lift-off, and maintaining the ability to easily assemble microbial communities. The design and fabrication was successfully demonstrated through the stochastic seeding and imaging of GFP expressing E. coli. Ultimately, this design will also provide more convenient fluidic access to the agar microwell lid allowing modulation of the chemical environment around the arrays in real-time. Future work will explore methods to integrate both microfluidics and microelectrode components into the transparent well platform.

\section{ACKNOWLEDGMENTS}

This work was funded by the Oak Ridge National Laboratory Lab Directed Research and Development (LDRD) program. The fabrication of the transparent microwell arrays was carried out in the Nanofabrication Research Laboratory at the Center for Nanophase Materials Sciences, which is a DOE Office of Science User Facility.

${ }^{1}$ R. H. Hansen, A. C. Timm, C. M. Timm, A. N. Bible, J. L. MorrellFalvey, D. A. Pelletier, M. L. Simpson, M. J. Doktycz, and S. T. Retterer, PLoS One 11, e0155080 (2016).

${ }^{2}$ A. E. F. Little, C. J. Robinson, S. B. Peterson, K. F. Raffa, and J. Handelsman, Annu. Rev. Microbiol. 62, 375 (2008).

${ }^{3}$ J. Zhou et al., PNAS 111, E836 (2014).

${ }^{4}$ F. J. H. Hol and C. Dekker, Science 346, 1251821 (2014).

${ }^{5}$ C. J. Ingham, A. Sprenkels, J. Bomer, D. Molenaar, A. van den Berg, J. E. T. V. H. Vlieg, and W. M. de Vos, Proc. Natl. Acad. Sci. U. S. A. 104, 18217 (2007).

${ }^{6}$ Y. Zhang, Y.-P. Ho, Y.-L. Chiu, H. F. Chan, B. Chlebina, T. Schuhmann, L. You, and K. W. Leong, Biomaterials 34, 4564 (2013).

${ }^{7}$ J. Q. Boedicker, L. Li, T. R. Kline, and R. F. Ismagilov, Lab Chip 8, 1265 (2008).

${ }^{8}$ K. Leung et al., Proc. Natl. Acad. Sci. U. S. A. 109, 7665 (2012).

${ }^{9}$ M. Lian, C. P. Collier, M. J. Doktycz, and S. T. Retterer, Biomicrofluidics 6, 044108 (2012)

${ }^{10}$ K. Churski, T. S. Kaminski, S. Jakiela, W. Kamysz, W. Baranska-Rybak, D. B. Weibel, and P. Garstecki, Lab Chip 12, 1629 (2012).

${ }^{11}$ Y.-J. Eun, A. S. Utada, M. F. Copeland, S. Takeuchi, and D. B. Weibel, ACS Chem. Biol. 6, 260 (2011).

${ }^{12}$ R. N. Orth, J. Kameoka, W. R. Zipfel, B. Ilic, W. W. Webb, T. G. Clark, and H. G. Craighead, Biophys. J. 85, 3066 (2003).

${ }^{13}$ C. P. Tan and H. G. Craighead, Materials 3, 1803 (2010).

${ }^{14}$ B. Ilic and H. G. Craighead, Biomed. Microdevices 2, 317 (2000). 